\title{
Thermodynamics of a black hole
}

\author{
K. A. I. L. Wijewardena Gamalath*, N. S. Rajapakse \\ Department of Physics, University of Colombo, Colombo 3, Sri Lanka \\ *E-mail address: imalie@phys.cmb.ac.lk
}

\begin{abstract}
A simple model was setup to find the mass variation over time for a Schwarzschild black hole. The temperature and entropy of a black hole was obtained from the numerically solved mass variation and the time variations of the black hole thermodynamic parameters were simulated. The mass of a given black hole reduces rapidly. The time taken for a black hole to vanish increases in an increasing rate with the given initial mass of the black hole. The temperature of a black hole drastically increases at the final stage of the black hole evaporation. The colour attributed to that temperature was found to be in the visible region for a significant amount of time. The black hole entropy also drastically reduces with its mass and through Hawking radiation it is added to the rest of the universe.
\end{abstract}

Keywords: Schwarzschild black hole; Hawking radiation; thermodynamic parameters; mass variation; entropy; temperature

\section{INTRODUCTION}

One of the central difficulties about the thermodynamics of gravitating systems is the apparent absence of true equilibrium. Unlike ordinary laboratory thermodynamic systems, a star is made hotter not by adding energy but by removing it. If the Sun were to suddenly lose all its heat energy, it would rapidly shrink to a fraction of its present size and, after a period of oscillation, take up a new condition at a much higher temperature. The ever-present threat of gravitational shrinkage under its own weight makes every star potentially unstable against catastrophe. The strength of gravity at its surface can be increased either if the star shrinks or if more mass is added. According to the general theory of relativity, gravity affects the properties of light and this is manifested in the behavior of light rays travelling radially outwards from the surface of a star. In order to escape from the star the light has to do work to overcome the surface gravity. Therefore its energy and hence frequency will be somewhat diminished giving rise to gravitational red shift. For more compact and massive objects, the red shift can become enormous. For a spherical, uncharged star if the radius of the star shrinks below the Schwarzschild radius, the light from its surface becomes redshifted without a limit, so that it can no longer be seen, it is black. The spherical surface with Schwarzschild radius acts as an event horizon, separating the events which can be viewed from a great distance from those which cannot, however long one waits. The region inside the horizon, once the star has shrunk away to nothing is empty and from the exterior universe, black and inaccessible. It is therefore 
called a black hole. The key to understanding black holes and especially their connection with thermodynamics is to appreciate the meaning of the so-called event horizon [1].

Schwarzschild black hole, a solution to the Einstein's field equations, plays an important role in understanding the general theory of relativity. Seeking into black hole mechanics is interesting as it establishes astonishing connections between general theory of relativity, quantum mechanics and statistical mechanics. Slightly perturbed equilibrium solutions of the Schwarzschild black hole give rise to the black hole dynamics and they turned out to have a close analogy to the conventional thermodynamic laws. Bekenstein [2] proposed that a suitable multiple of the area of the event horizon of a black hole should be interpreted as its entropy. The idea that a black hole has entropy was first noticed by Floyd and Penrose [3] from the realization that its event horizon surface area exhibits remarkable tendency to increase when undergoing any transformation. The Hawking radiation was postulated by Hawking et al. $[4,5,6]$ and they gave a general proof that the surface area of the black hole cannot decrease in any process. By treating the space time curvature at the event horizon of a black hole through quantum field theory, Hawking [7] showed that the black hole should emit particles with a characteristic black body spectrum of a temperature, later named as Hawking temperature. At the purely classical level, black holes in general relativity obey certain laws which bear a remarkable mathematical resemblance to the ordinary laws of thermodynamics. A black hole emits particles with the characteristic spectrum and loose mass and energy due to the Hawking radiation through a quantum mechanical process and at the same time gain energy and mass from the cosmic microwave background radiation.

In the present work considering the surface of the event horizon of a Schwarzschild black hole as a perfect black body, a simple model was obtained for the dynamic relationship between these two phenomena. To solve the differential equation for the mass variation of a black hole, the Euler numerical method for solving the linear first order differential equations was employed. Using the numerical solution, the variations of the thermodynamic parameters were simulated. At first, the cosmic microwave background (CMB) radiation was considered as a constant throughout the time period considered. But the time span of the simulations turned out to be very much larger than the present age of the universe. Therefore, the variation of the cosmic microwave background radiation with time in the present era which is the dark matter dominant era of the universe was considered and integrated into the previously formed differential equation for the mass variation.

\section{THERMODYNAMICS OF A BLCK HOLE}

Black holes are among the most fascinating things in the universe. When Hydrogen is totally consumed in the fusion reaction in a star, it starts to collapse due to its own gravitational field. The internal temperature and pressure are increased as a result and heavier element fusion reactions are then ignited. The newly introduced radiation pressure will balance the gravitational pressure. When all elements

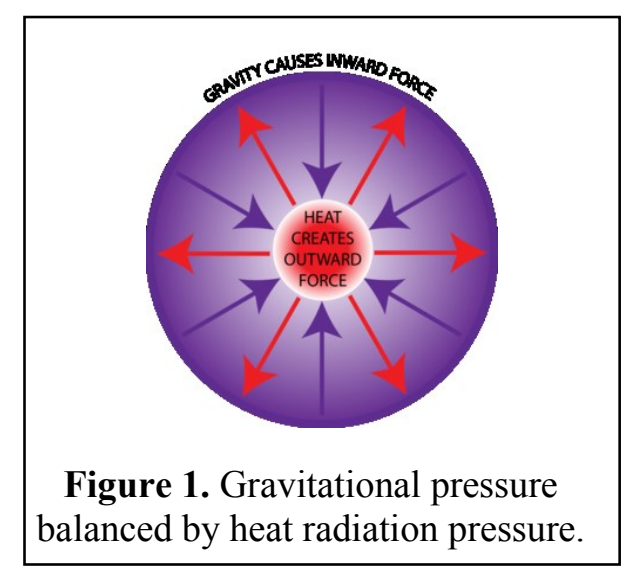
up to iron get fused, no fusible fuels will be left and fusion reactions will be terminated. This will be followed by the termination of radiation pressure and there will be nothing to 
prevent the star from collapsing into its ultimate fate, causing an explosion known as Supernova. In this explosion, some of the star dust will be sent out in to the universe which will eventually contribute to later generations of stars and planets and the remainder will be collapsed towards it centre due to gravity and its ultimate fate will be determined by the mass of the original star. Stars with masses bellow 1.3 times the solar mass $M_{\mathrm{e}}$ will end up as white dwarfs while stars with masses in between 1.3 and 3 times the solar mass will end up as neutron stars. Stars with masses above 3 times the solar mass will further collapse into a singularity with infinite density ending up as black holes [8].

A black hole is immensely dense and its escape velocity is even higher than the velocity of light. The boundary at where the escape velocity equals the velocity of light is the event horizon, a hypothetical sphere or an ellipsoid depending on whether the black hole is having an angular momentum or not. Beyond the event horizon of a black hole all the known physical laws are no longer valid and one needs a quantum theory of gravity to seek in to the ultimate singularity at the centre of the black hole [7]. Accordingly to the No-Hair theorem a black hole is uniquely determined by three parameters, its mass, charge and angular momentum [9]. All types of black holes carry a mass. Schwarzschild black hole does not carry a charge no angular momentum. Another way of categorizing black holes is by their mass. Although fine boundaries cannot be defined physicists consider black holes under four classes depending on their mass, super massive $\left(\sim 10^{5}-10^{9} M_{\mathrm{e}}\right)$, intermediate mass $\left(\sim 10^{3} M_{\mathrm{e}}\right)$, stellar $\left(\sim 10 M_{\mathrm{e}}\right)$ and micro (up to $\sim M_{\text {Moon }}$ ) black holes [10].

Schwarzschild black hole is the simplest black hole existing and characterized only by its mass. This simple black hole is astronomically, a collapse of an uncharged and nonrotating star with spherical symmetry. The geometry of a spherically symmetric vacuum, i.e. vacuum space-time outside the spherical black hole is the Schwarzschild geometry describable in terms of the Schwarzschild metric [9]:

$$
d s^{2}=\left(1-\frac{r_{s}}{r}\right) d t^{2}-\left(1-\frac{r_{s}}{r}\right)^{-1} d r^{2}-r^{2}\left(d \theta^{2}+\sin ^{2} \theta d \phi^{2}\right)
$$

where the Schwarzschild radius $r_{s}$ is given by

$$
r_{s}=\frac{2 G m}{c^{2}}
$$

here $G$ is the universal constant of gravitation, $m$ is the mass of the black hole and $c$ is the velocity of light in vacuum. Event horizon, a hypothetical sphere surrounding the black hole with a radius equal to Schwarzschild radius marks the so called point of no return as at this boundary the escape velocity reaches the value of the velocity of light.

There is a close relationship between the traditional thermodynamic parameters and black hole parameters. That is, the internal energy $E$ to mass $m$ of the black hole, temperature $T$ to surface gravity $\kappa$ of the event horizon and the entropy $S$ to the area $A$ of the event horizon [4]. Having assigned black hole parameters correctly to the analogous parameters in thermodynamics, the, zeroth, first and third laws of classical thermodynamics can be re-interpreted for a black hole [4]. If area $A$ of the event horizon plays the role of entropy, then surface gravity $\kappa$ plays the role of temperature 
$(\kappa d A \sim T d S)$ The surface gravity defined locally, is always constant over the event horizon of a stationary black hole. This constancy is reminiscent of the zeroth law of thermodynamics which states that the temperature is uniform everywhere in a system in thermal equilibrium. Considering a quasi-static process in which a small mass $d m$ is added to a black hole, the entropy term in the first law is $d m=\kappa d A / 8 \pi G$. For a Schwarzschild black hole $\partial m / \partial A=\kappa / 8 \pi G$. This is really just an expression of mass-energy conservation and corresponds to the first law. Cosmic censorship implies the unattainability of absolute zero, $\kappa=0$. Therefore third law states that the surface gravity of the horizon cannot be reduced to zero in a finite number of steps [10].

Because gravity always attracts, there is a general tendency for self-gravitating systems to grow rather than shrink. The inability for light to emerge from inside the event horizon of a black hole precludes the escape of any material and the event horizon acts as an asymmetric one-way surface. This indicates the second law of thermodynamics, in which there is an asymmetric tendency for a one-way increase in entropy. The size of the black hole is analogous to the entropy. It keeps on increasing. This analogy is almost trivial for a spherical, electrically neutral Schwarzschild black hole. A given global, or macro, state can be realized by an enormous number of internal microstates. This suggests that black holes have very high entropy, representing in some sense the maximum entropy, equilibrium end state of gravitational collapse. Hawking [5] has shown in a theorem that the event horizon area cannot decrease in any process, so long as locally negative energy which gravitates repulsively is not involved. This strong analogy between the event horizon area and the entropy led to the use of the name second law in connection with Hawking's area theorem, stating that the horizon area can never decrease assuming cosmic censorship and a positive energy condition $d A \geq 0$ [5]. Equality corresponds to reversibility. The laws of classical thermodynamics and their analogues for a black hole are tabulated in table 1.

Table 1. Thermodynamics laws interpreted for a Schwarzschild black hole [11].

\begin{tabular}{|c|c|c|}
\hline Law & In thermodynamics & For a black hole \\
\hline Zeroth law & $\begin{array}{c}\text { Temperature is uniform in a } \\
\text { thermodynamic system in } \\
\text { equilibrium }\end{array}$ & $\begin{array}{c}\text { Surface gravity throughout } \\
\text { the event horizon is uniform }\end{array}$ \\
\hline First law & $d E=T d S+$ work terms & $d m=\frac{\kappa}{8 \pi G} d A+$ work terms \\
\hline Second law & $T=0$ & $\kappa=0$ \\
Third law & $\begin{array}{c}\text { Cannot be achieved within a } \\
\text { finite number of cycles }\end{array}$ & $\begin{array}{c}\text { Cannot be achieved within a } \\
\text { finite number of cycles }\end{array}$ \\
\hline
\end{tabular}

A classical black hole is a non-quantum black hole which emits neither matter nor radiation. But when the thermodynamic analogy is taken into account, a few oversights of 
the black hole thermodynamics became apparent: the temperature of a black hole vanishes; in natural units, the entropy is dimensionless whereas that of the event horizon, the area is length squared; the event horizon area of each black hole individually is nondecreasing whereas only the total entropy is non-decreasing in thermodynamics [12]. To overcome these, a quantum black hole was introduced.

Due to the vacuum fluctuations and Heisenberg uncertainty principle of energy and time, particles and anti-particles are constantly created and annihilated in the free space. At the vicinity of the event horizon of a black hole, the anti-particle can fall into the black hole leaving the particle free. The matter particles will not be subjected to annihilation but tunnelled through the universe and can be observed by an outside observer. The particle will be identified as if it had been emitted by the black hole itself. This radiation is known as the Hawking radiation. Therefore in a quantum black hole, its mass is lost through Hawking radiation [1]. Around a massive,

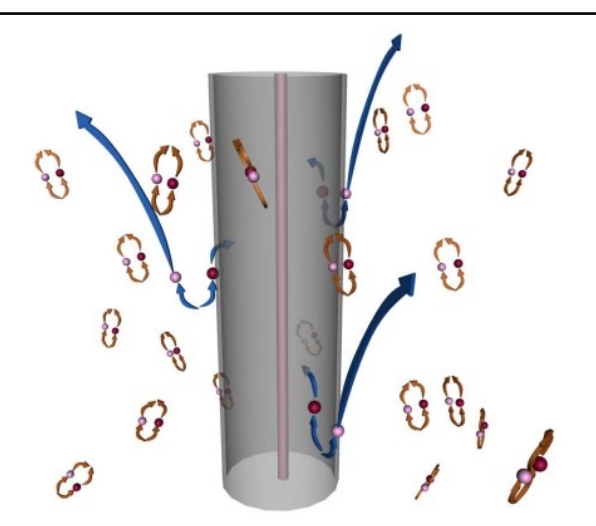

Figure 2. Hawking radiation. static body, the gravitational field or space curvature induces a static vacuum stress, which is rather like the vacuum polarization produced by the Coulomb field of a nucleus which contributes to the Lamb shift. This vacuum stress can be envisaged as a cloud of negative energy surrounding the body and falling off rapidly in density at large distance [13]. The presence of a cloud of negative vacuum energy can become intense near a black hole and comparable in magnitude to the energy of the Hawking radiation. When the star implodes, a hole appears in the centre of the negative-energy cloud, so this negative energy continuously streams into the black hole as the Hawking radiation streams away to infinity. Conservation of energy demands that the Hawking flux is paid for by this ingoing negative energy and the effect is to steadily reduce the mass-energy of the black hole at just the right rate to compensate for the thermal radiation appearing in the surroundings. In this way the mass of the hole supplies the energy of the Hawking radiation, not by this energy emerging from inside the hole which is impossible because of the event horizon but by receiving negative mass-energy from the inflowing vacuum stream. Thus the requirements of both causality and energy conservation are simultaneously satisfied. As the mass of the hole is steadily reduced it shrinks in size. The violation of Hawking's area theorem is explained by the presence of negative energy. From the thermodynamic point of view there is also consistency: the entropy of the black hole measured by its area diminishes, but the creation of high entropy thermal radiation in the surrounding environment more than offsets this decrease and saves the second law of thermodynamics. So long as the rate of shrinkage of the hole is much less than the frequency of the Hawking radiation, the effect of the back-reaction on the radiation spectrum can be ignored and the black hole still behaves as if it is approximately in thermodynamic equilibrium [1].

The black hole radiation is thermal in nature, therefore generates a rise in entropy in the surrounding region. The generalized entropy $S^{\prime}$ was introduced by Bekenstein [14] to account for this sort of entropy. It is defined as the sum of the black hole's entropy $S_{b h}$ and the entropy of the surrounding matter $S_{\mathrm{s}}$, i.e. $S^{\prime}=S_{b h}+S_{s}$. This statement is known as the Generalized second law $\Delta S^{\prime} \geq 0$. A quantum black hole has a non-zero temperature [12]. 
A black hole has a Hawking temperature proportional to the Planck constant [6]. The entropy is one fourth the horizon area divided by the plank length squared. The area and hence the entropy of an individual black hole can be decreased through Hawking Radiation. Hawking temperature $T_{H}$ for a Schwarzschild black hole is given by the expression [1]:

$$
T_{H}=\frac{\hbar \kappa}{2 \pi k_{B}}=\frac{\hbar c^{3}}{8 \pi G k_{B} m}
$$

where $\hbar$ is the reduced Planck constant, $G$ is the universal constant of gravity, $m$ is the black hole mass and $k_{B}$ is the Boltzmann constant. In order for the second law of thermodynamics not to be violated, a black hole should possess non zero entropy [2]. The entropy of a black hole is sometimes called Bekenstein-Hawking entropy. It is proportional to the surface area $A$ of its event horizon and is given by the following formula [6]:

$$
S_{b h}=\frac{k_{B} A}{4 G \hbar}=\frac{k_{B} \pi r_{s}^{2}}{G \hbar}
$$

The present era of the universe is dark matter dominated. Hence the cosmic microwave background temperature decays in an exponential manner. The temperature variation is given by the following expression [7]:

$$
T(t)=2.7 e^{-H t}
$$

where $H$ is the Hubble's constant with a value $H=69.32 \pm 0.80 \mathrm{~km} / \mathrm{s} / \mathrm{Mpc}$ and $t$ is the time measured from the present. The power per unit area $I$ emitted can be written as

$$
I=\frac{\pi^{2} k_{B}^{3}}{60 \hbar^{2} c^{2}} T^{4}=\sigma T^{4} \quad\left(\sigma=5.67 \times 10^{-8} W^{-2} K^{-4}\right) .
$$

If a black body at temperature $T$ is in a heat bath at temperature $T_{0}$, then the energy is emitted from both the black body and the surrounding according to their own temperatures. Hence the energy is simultaneously lost and added to the black body. Then the net total power $P$ emitted by the black body can be stated as follows:

$$
P=A \sigma\left(T^{4}-T_{0}^{4}\right)
$$

\section{CRITICAL MASS}

A black hole regardless of its type can be considered as a black body emitting a radiation spectrum with a characteristic temperature, Hawking temperature $T_{\mathrm{H}}$. At the same time it is in the thermal bath of so called cosmic microwave background (CMB) 
radiation. Hence energy is simultaneously gained and lost by the black hole. This situation can be mathematically modelled by the Stephan-Boltzmann law:

$$
-\frac{d E(t)}{d t}=\sigma A\left(T_{H}^{4}(t)-T_{S}^{4}(t)\right)
$$

where $E$ is the total energy of the black hole, $A$ is the surface area of the black body and $T_{S}$ is the surrounding temperature. From equation 3, the Hawking temperature can be written as

$$
\mathrm{T}_{\mathrm{H}}=\frac{\alpha}{\mathrm{m}}, \quad\left(\alpha=\frac{\mathrm{hc}^{3}}{8 \pi \mathrm{Gk}_{\mathrm{B}}}\right)
$$

Energy of an isolated system in general relativity can be defined as the gravitating mass as measured at infinity times $c^{2}$. This energy, which is the numerical value of the Hamiltonian that generates the time translation symmetry at infinity, is a conserved quantity. Positivity of the total energy at infinity does not necessarily mean that the system cannot radiate an infinite energy while collapsing, since both the energy of the radiation and the energy of the leftover system are included in the total energy. According to the special theory of relativity, the change in mass is related to change in energy by,

$$
\frac{d m}{d t}=\frac{1}{c^{2}} \frac{d E}{d t}
$$

Using the relations given in equations 9 and 10 in 8 :

$$
-\frac{d m(t)}{d t}=\frac{1}{c^{2}} \sigma A\left(\frac{\alpha^{4}}{m^{4}}-T_{S}^{4}(t)\right)
$$

The area of the event horizon of a Schwarzschild black hole is

$$
A=4 \pi r_{s}^{2}=\frac{16 \pi G^{2} m^{2}}{c^{4}}=\beta m^{2} \quad\left(\beta=\frac{16 \pi G^{2}}{c^{4}}\right)
$$

With equations 9 and 12, equation 11 can be written as:

$$
\frac{d m}{d t}=\left(\frac{\sigma \beta}{c^{2}}\right)\left(T_{S}^{4}(t) m^{2}-\frac{\alpha^{4}}{m^{2}}\right)=\left(\frac{16 \pi G^{2} \sigma}{c^{6}}\right) T_{S}^{4}(t) m^{2}-\left(\frac{\hbar}{256 \pi^{3} G^{2} k_{B}^{4}}\right) \frac{1}{m^{2}}
$$

Substituting the values of the constants, equation 13 reads,

$$
\frac{d m}{d t}=\left(1.776 \times 10^{-77}\right) T_{S}^{4}(t) m^{2}-\left(3.914 \times 10^{15}\right) \frac{1}{m^{2}} .
$$


Defining the mass of the black hole with respect to the solar mass $M_{\mathrm{e}}$, the parameter $\eta=m / M_{\mathrm{e}}$ was introduced. Equation 14 in terms of $\eta$,

$$
\frac{d \eta}{d t}=\left(3.534 \times 10^{-47}\right) T_{S}^{4}(t) \eta^{2}-\left(4.967 \times 10^{-76}\right) \frac{1}{\eta^{2}}
$$

Accordingly to the Einstein's special theory of relativity there is a direct connection between mass and energy while the thermodynamic counterpart for energy for a black hole is mass. Hence in the thermodynamic point of view, the mass of a black hole is identified as its internal energy. Mass variation with time can be obtained by numerically solving the differential equation given in equation 15. Initially, the background radiation of the universe was assumed to be a constant throughout the time. This constant cosmic microwave background (CMB) radiation temperature was taken as $2.7 \mathrm{~K}$ and is a good enough assumption, if the life time of the black hole is appreciably less compared to the temperature variation time of the universe. The equation 15 was solved numerically for a black hole with an initial mass $\eta=2.3 \times 10^{-8} \quad$ with radiation temperature $T_{s}(t)=2.7 K$. The mass variation is shown in the figure 3(a). As mass is gained by the black hole, the surface area of its event horizon expands

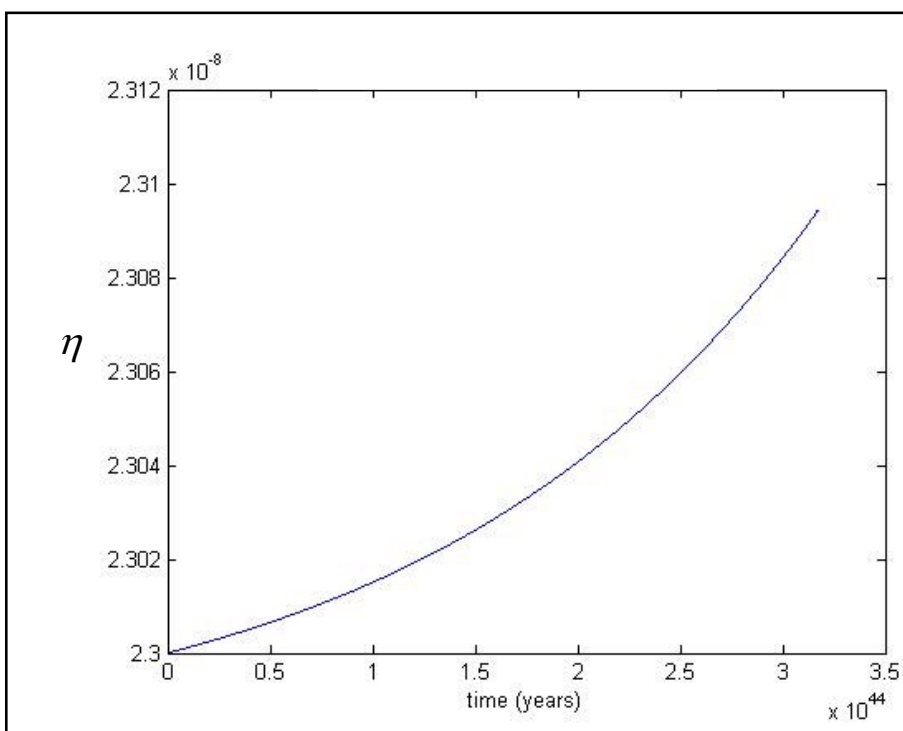

Figure 3(a). Mass variation for initial mass $\eta=2.3 \times 10^{-8}$ black hole- 1 .

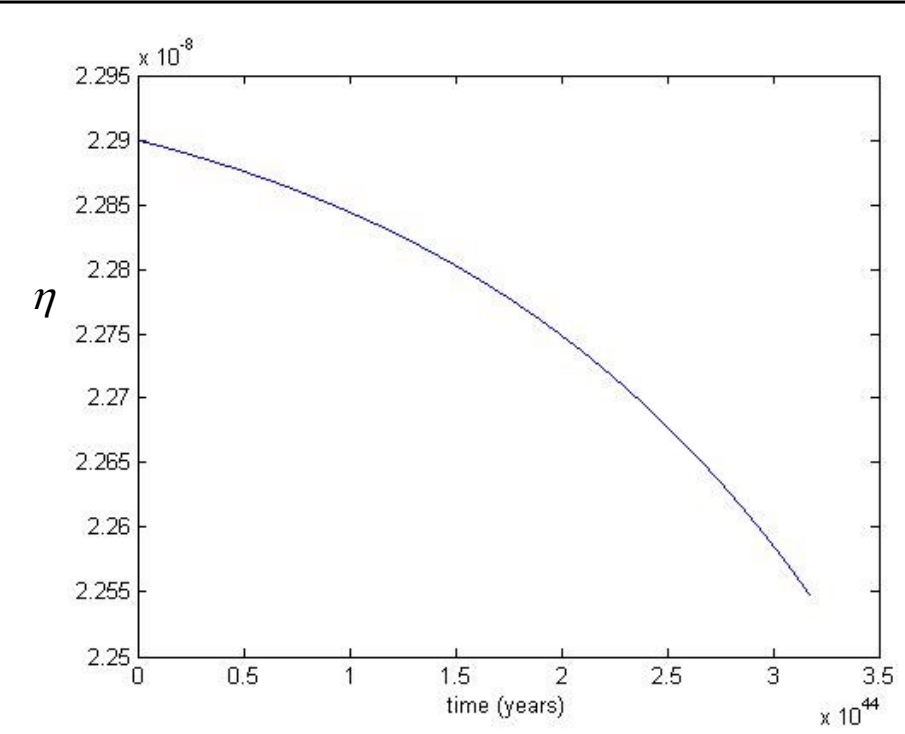

Figure 3(b). Mass variation for initial mass $\eta=2.29 \times 10^{-8}$ black hole- 2 . increasing the area of the interface between the black hole and the universe allowing the black hole to absorb more and more radiation. Thereby the energy from the universe causes it to grow even faster and the mass increases at an increasing rate. Meanwhile the increment of the mass reduces the Hawking radiation, causing the black hole to lose mass at a lower and lower rate. In the figure $3(b)$ the mass variation of a black hole with an 
initial mass $\eta=2.29 \times 10^{-8}$ is shown. In this case the mass is lost by the black hole at an increasing rate. As the mass is lost by the black hole, it shrinks causing the surface area of its event horizon to be reduced. And hence lesser radiation flux is absorbed by the black hole causing its mass adding rate to be diminished. Meantime the Hawking radiation becomes more prominent and that causes the black hole to evaporate at an increasing rate.

The first time derivative of the mass switches the sign at a critical point somewhere near $\eta=2.3 \times 10^{-8}$. The critical mass can be found by evaluating the roots of the first time derivative of the mass of the black hole. A graphical representation of the procedure is given in figure 4. The critical mass of the stationary black hole is $\eta=2.297867 \times 10^{-8}$. At this critical mass, the rate of which the radiation or mass is received by the black body from the cosmic microwave background radiation is equal to the rate at which it loses mass through the Hawking radiation.

Figure 5(a) shows the simulation of time variation of the mass of a black hole with masses well below that of the critical value for constant CMB temperature. The present age of the universe which is approximately 13.7 billion years is denoted by the red vertical line. Hence the black holes with masses less than $\eta=8.0 \times 10^{-20}$ which prevailed at the very early stages of the universe must be totally evaporated by the present time. The range of time of consideration is in billions of years which is even more than the present age of the universe. Hence the assumption that the CMB temperature is constant was not

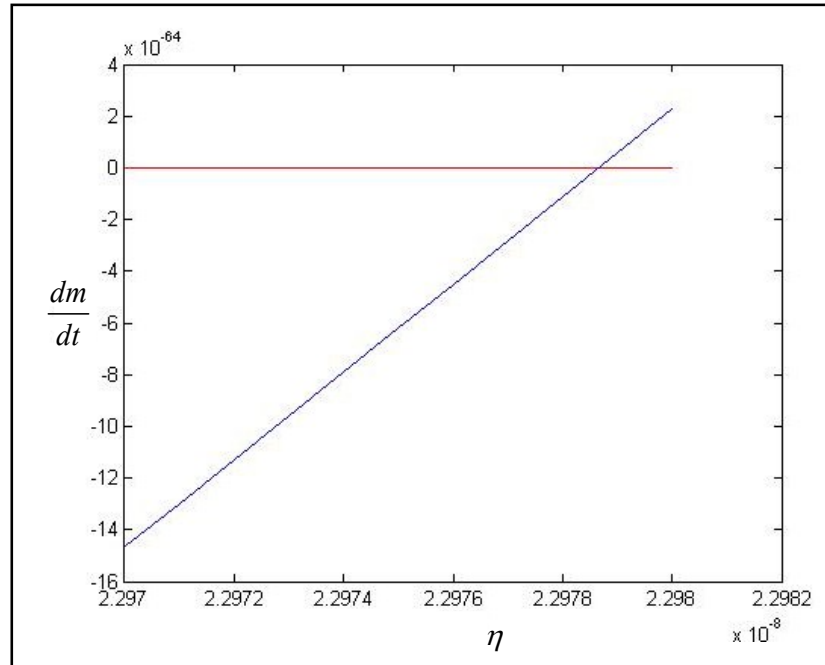

Figure 4. First time derivative of the mass.

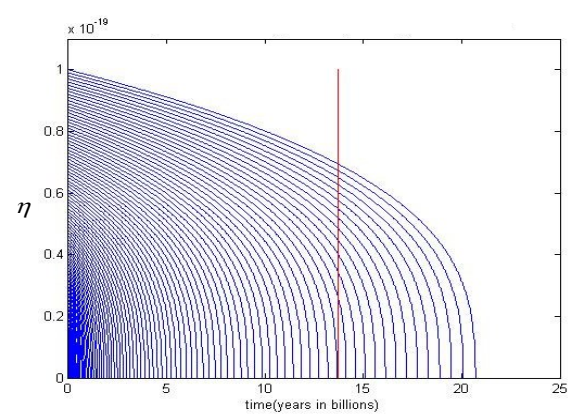

Figure 5(a). Life span of the black holes against the present age of the universe with constant CMB temperature.

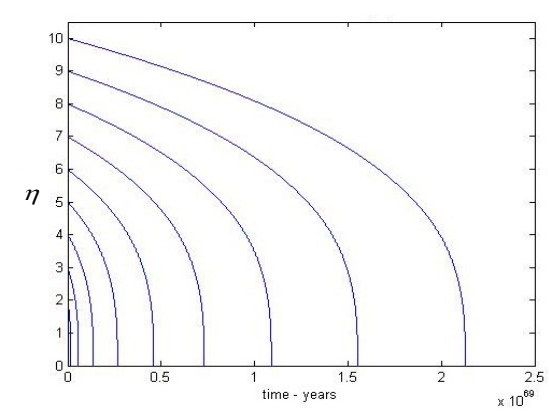

Figure 5(b). Life span of the black holes against the present age of the universe with varying CMB temperature. valid. The present era of the universe is dark matter dominated and the CMB temperature decay exponentially according to the equation 5 . Figure $5(b)$ shows the simulation of time variation of the mass of a black hole with masses well below that of the critical value for varying $\mathrm{CMB}$ temperatures. The time taken by a ten solar mass black hole to get totally 
evaporated is extremely large when compared to the present age of the universe but is still finite. Hence no black hole is stable regardless how massive it is and finally will be completely evaporated.

A mass range of a relatively, short lived black holes was used to study the behaviour of the vanishing time of black holes. The stimulation for different masses is shown in figure 6 . The time taken by a black hole increases at an increasing rate with the black hole mass. This was verified by plotting the vanishing time against the initial black hole mass and shown in figure 7 . The vanishing time increases at an increasing rate with the initial

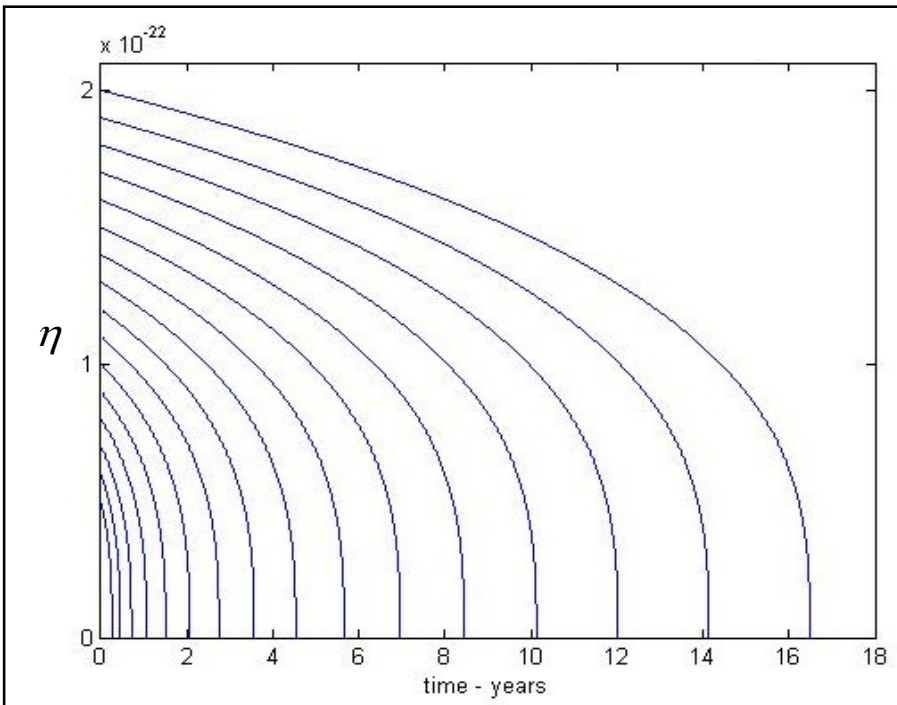

Figure 6. Mass variation with constant universe temperature for short lived black holes. mass of the black hole. The variation was fitted into a polynomial curve.

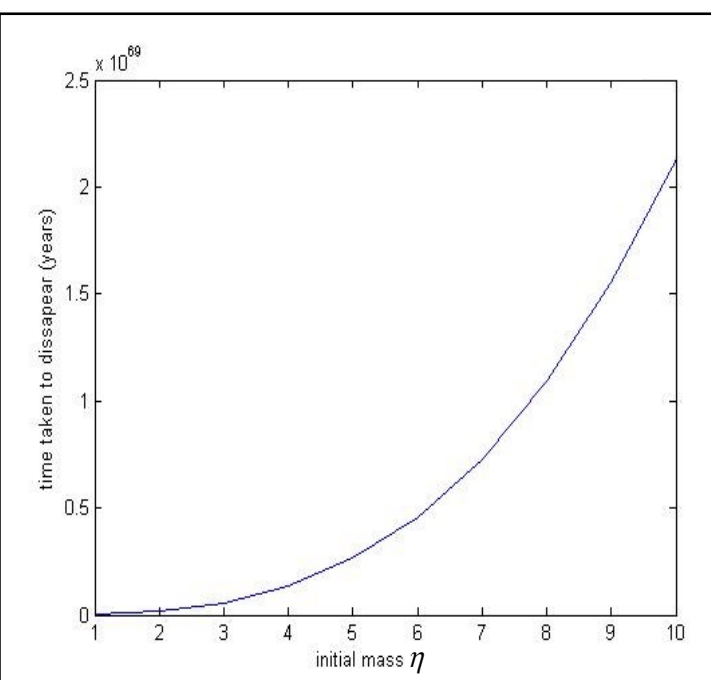

Figure 7. Vanishing time against the initial mass.

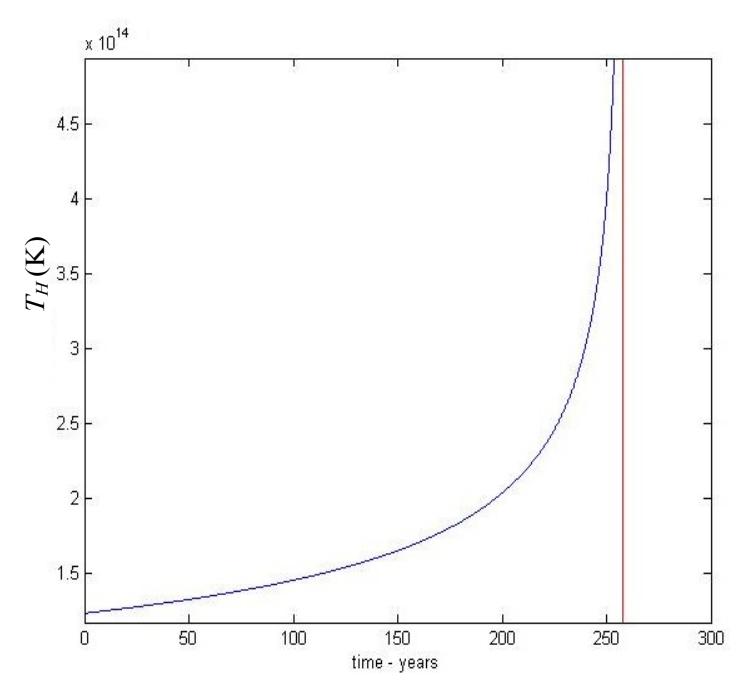

Figure 8. Temperature variation of a black hole $\eta=5 \times 10^{-22}$ at constant CMB over time.

\section{TEMPERATURE}

For a Schwarzschild black hole, the only characteristic determinant is its mass. Therefore the temperature can be written in terms of its mass. The Hawking temperature given in equation 3 can be modified as follows: 


$$
T_{H}=\frac{\hbar c^{3}}{8 \pi G k_{B} M_{\mathrm{e}}} \frac{1}{\eta}
$$

From the above
equation, using the mass
variation of black hole over the time, the temperature variation was obtained. The figure 8 demonstrates the simulation of the temperature variation over time for a black hole of mass $\eta=5 \times 10^{-22}$ at constant $\mathrm{CMB}$ by the blue curves. They are asymptotic to the red line where the black hole vanishes. The figure 9 shows the temperature variation over time for a given mass range of black holes. The direction of the mass increment is shown by the marked arrow. And the red line asymptote is the time at which the black hole with the largest mass vanishes.

According to the Wien displacement law, a black body has a wavelength $\lambda_{\max }$ at which the maximum amount of radiation is carried out from the black body. This wavelength can be obtained by differentiating the Planck's

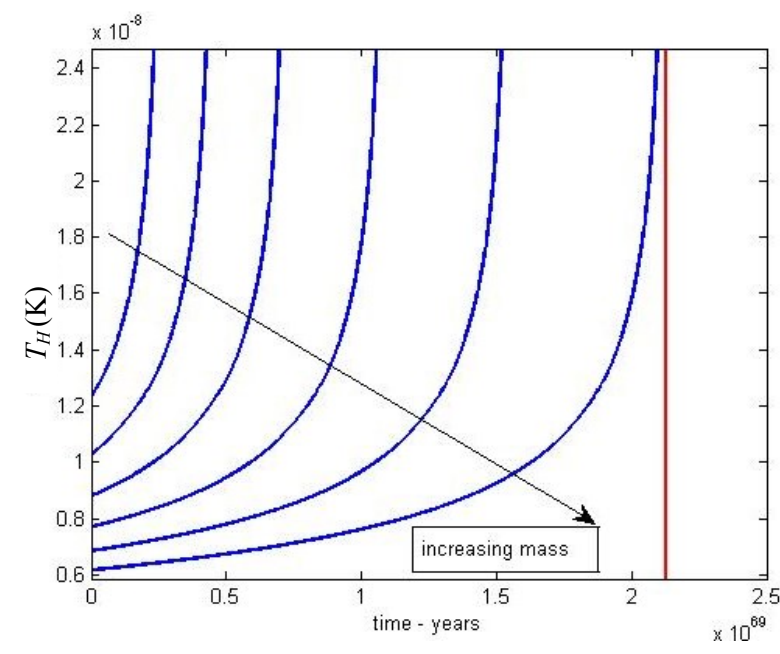

Figure 9. Temperature over time for a mass range of a black hole.

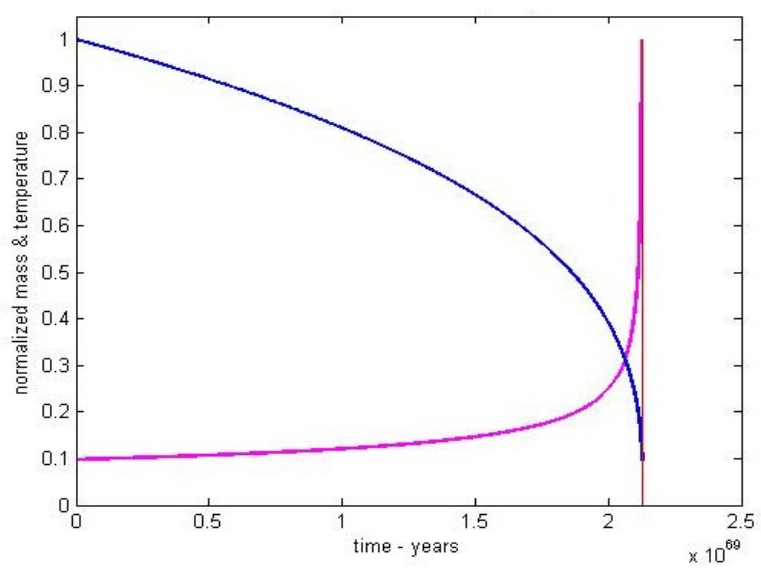

Figure 10. Normalized mass and temperature at constant CMB. black body energy distribution and setting it to zero.

$$
\lambda_{\max }=\frac{h c}{x k_{B} T}=\frac{2.8978 \times 10^{6}}{T} \mathrm{~nm}
$$

where $x=h c / \lambda k_{B} T$. From numerical methods the value of $x$ obtained for maximum radiation density is $x=4.965$. The simulation of the normalized mass and the normalized temperature at constant $\mathrm{CMB}$ is shown in the figure 10. Since the magnitudes of the mass and the temperature of the black hole are in different order, in order to compare the graph, the normalization concept that each curve divided by the its own maximum value was introduced. The normalized mass is represented by the blue curve while the normalized temperature is defined by the pink curve. This figure shows the inverse relation of the 
temperature of a black hole with its mass. The figure 11 shows the temperature variation over time for a range of masses of evaporating black holes in a 3D plot.
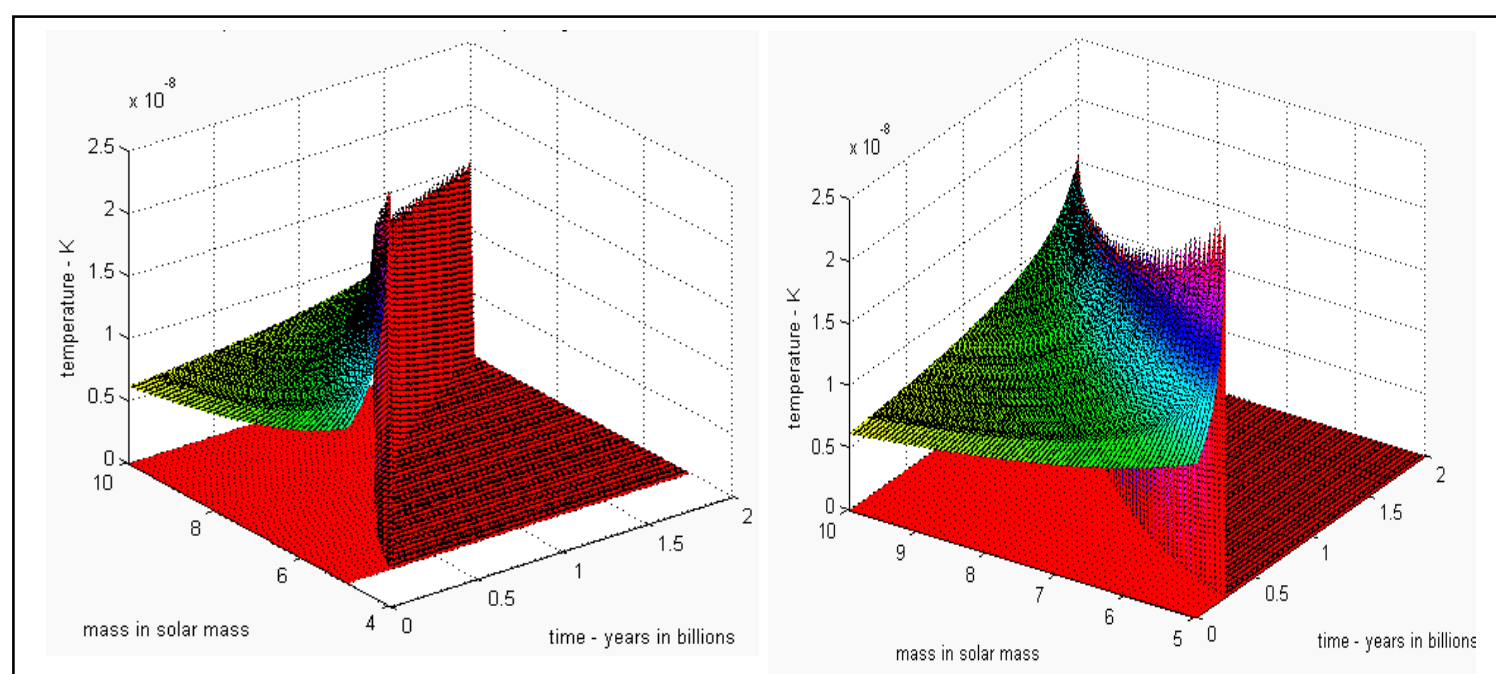

Figure 11. Temperature variation over time of evaporating black hole 1 (left) and 2 (right).

A unique colour can be attributed to the Hawking temperature of a black hole which is called as Hawking colour. From the equations 16 and 17 the maximum wavelength is,

$\lambda_{\max }=\left(4.693 \times 10^{4}\right) \eta \mathrm{mm}$

The simulation of the colour variation over time of a black hole at constant $\mathrm{CMB}$ is shown in figure 12 . The colour variation is represented by the blue curve and the range of the visible region is denoted by the two red horizontal lines. During

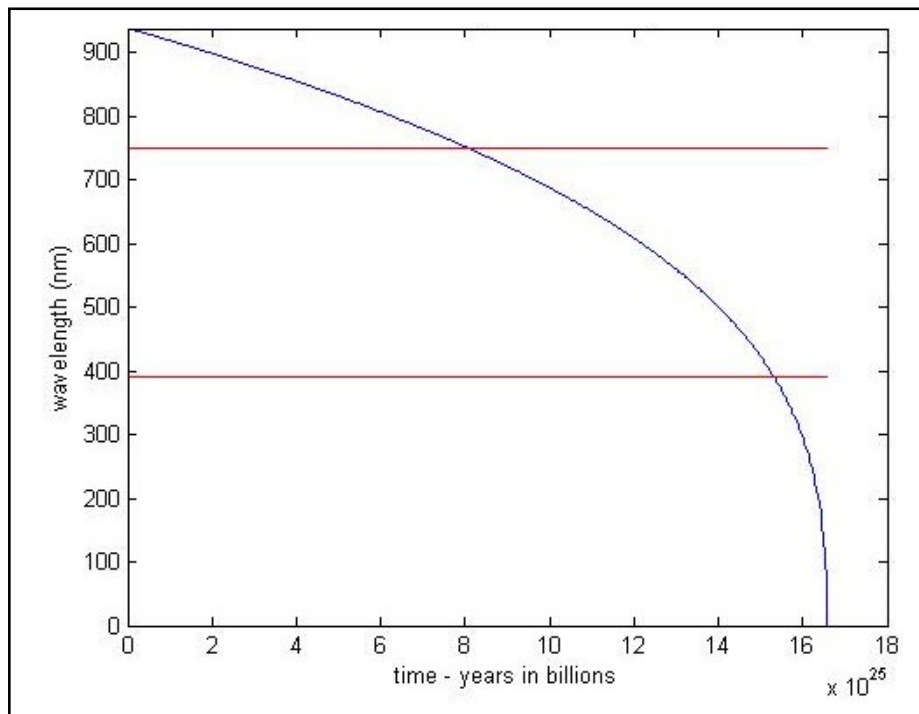

Figure 12. Colour variation of a black hole with time at constant $\mathrm{CMB}$.

the evaporation process, the black hole spends a significant amount of time in the visible region.

\section{ENTROPY}

For a Schwarzschild black hole, the entropy given in equation 4 with area obtained in equation 12 read 


$$
S_{b h}=\frac{k_{B} A}{4 G \hbar}=\left(\frac{4 \pi k_{B} G M_{s}^{2}}{\hbar c^{4}}\right) \eta^{2}
$$

The entropy variation over time of a black hole with varying cosmic microwave background temperature was simulated by taking the variation of equation 19 with equation 13. The simulation of entropy variation for different black hole masses is depicted in the figure 13. The direction of increasing mass is marked by the arrow. As the entropy of a black hole is directly proportional to the square of its mass, the entropy drops rapidly with the decreasing of mass. The normalized entropy and mass variation over time of a black hole is shown in Figure 14.

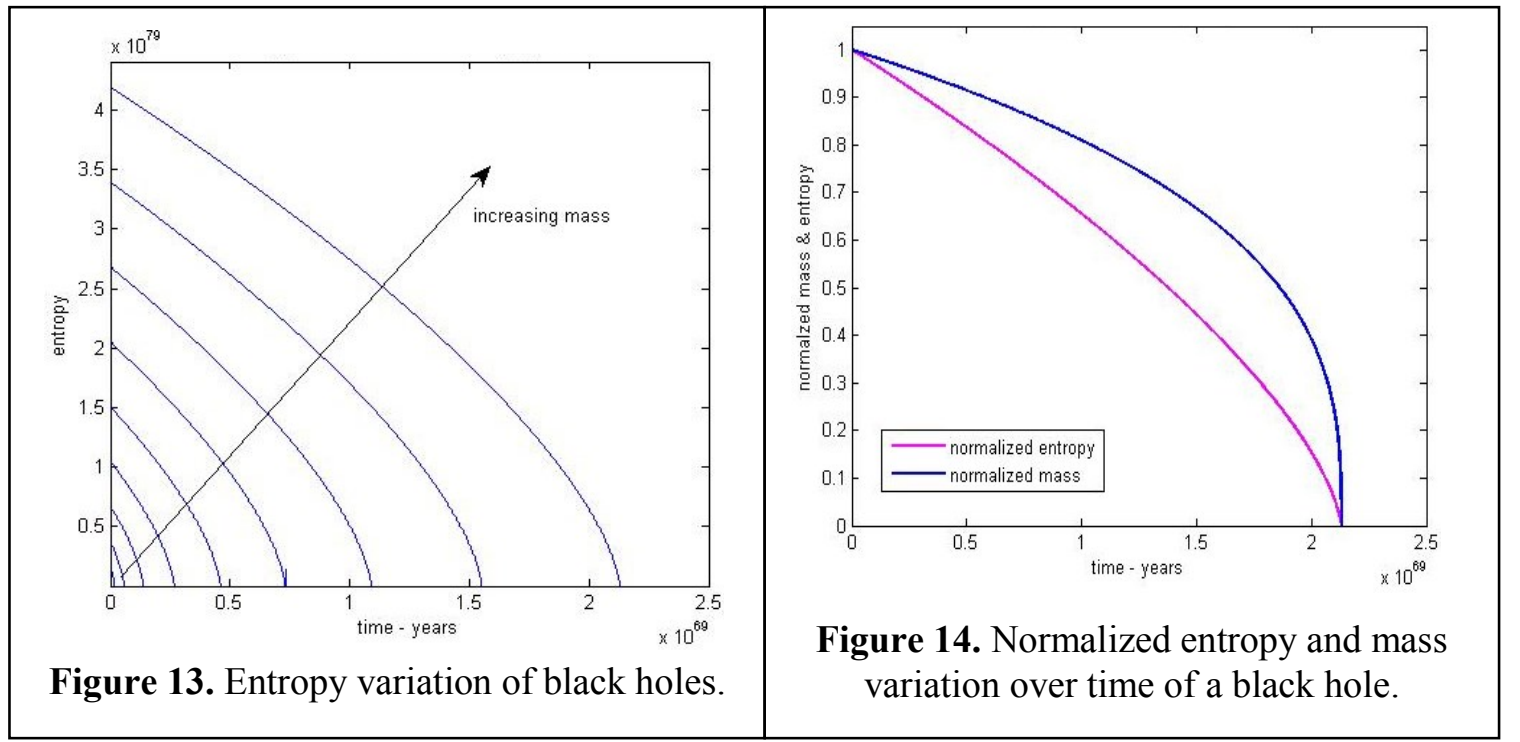

The simulation of entropy variation over time for a range of black holes masses, in three dimensions is shown in figure 15. The black holes with a larger mass initially possess a huge amount of entropy and with time as the mass decreases their entropy is also decreased. Even though during the evaporation process, the entropy of the black hole $S_{b h}$ is decreased, that entropy is carried away to the universe by the Hawking radiation.
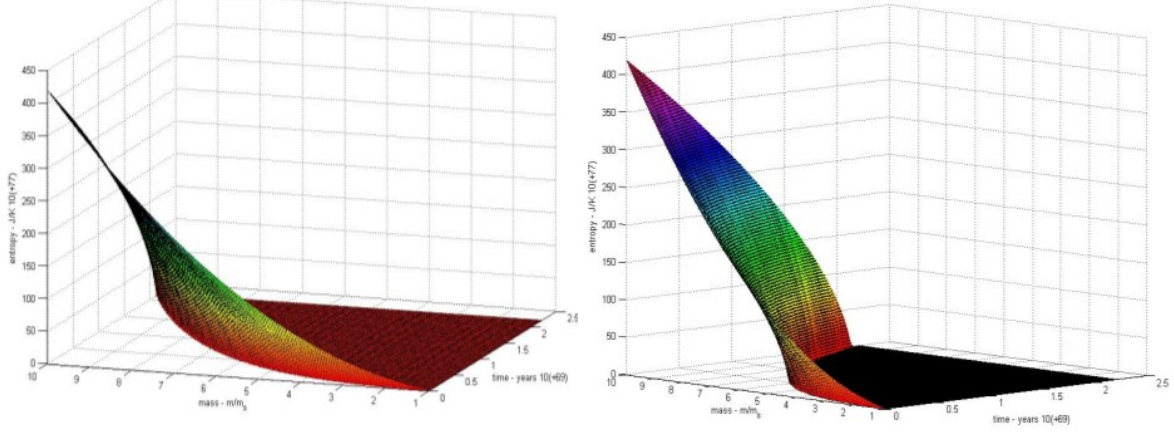

Figure 15. The simulation of entropy variation over time of black holes in a 3D plot for two elevations. 
Hence the total entropy of the universe is not decreased. That is the summation of the entropies of a black hole and its surrounding $S_{S}$ does not decrease with time i.e. $\Delta S_{b h}+\Delta S_{S} \geq 0$.

\section{CONCLUSION}

By extending the concepts of thermodynamics to include Schwarzschild black hole region, the thermodynamics laws may be generalized with remarkable ease to encompass these situations. The mathematical model assuming that energy is simultaneously gained and lost by the black hole according to the Stephan-Boltzmann law showed that, the mass of a black hole is steadily reducing with an increasing rate. The time taken for a black hole to vanish increased rapidly with its initial mass. With the constant cosmic microwave background temperature assumption, a critical mass for a black hole was determined at which the black hole is in dynamic equilibrium with the surrounding. The method is valid for short period of times. However in the dark matter dominant present era the cosmic microwave background temperature drops exponentially and no black hole can withstand the evaporation. The temperature of a black grows rapidly as it is evaporated and finally tends to infinity and vanishes in a very short period of time causing gamma ray bursts. Through the Wien displacement law a colour was attributed to a black hole and through the simulations it was observed that in the evaporation process a black hole spends a significant amount of time within the visible region. However even though such a black hole emits radiation in the visible region, the size of the surface area of such a black hole is too small to be noticed. The entropy is decreases rapidly with the black hole mass and through the Hawking radiation, the entropy is added to the rest of the universe. These simulations show that the four laws of thermodynamics can be adopted by this model to explain the dynamics of a black hole. The most attractive feature of black holes is that they enlarge our notion of thermodynamics.

\section{References}

[1] P.C.W Davies, Rep. Prog. Phys 41 (1978) 1313-1355.

[2] J. D. Bekenstein, Phys. Rev. D 7 (1973) 2333-2346.

[3] R. Penrose, R. M. Floyd, Nature 229 (1971) 177.

[4] J. M. Bardeen, N.Carter, S. W. Hawking, Commun. Math. Phys. 31 (2) (1973) 61170.

[5] S. W. Hawking, Phys. Rev. Lett. 26 (1971)1344.

[6] S. W. Hawking, 199, Nature (London) 248 (1974) 30. Commun. Math. Phys. 43 (1975)

[7] J. B. Hartle, S. W. Hawking. Phys.Rev. D 28 (12) (1983) 2960

[8] R.L. Piccioni, Einstein for everyone, (Jaico Publishing House, India, 2010).

[9] R.M.Wald, The Thermodynamics of Black Holes, Lecture Note, (University of Chicago ) 
[10]T. Jacobson, Phys. Rev. Lett. 75 (1995) 1260-1263. Introductory Lectures on Black Hole Thermodynamics (University of Utrecht, 2001).

[11]A. Dabholkar, S. Murthy, D. Zagier, JHEP 4 (2004) 015E.

[12]E. Poisson, A relativist's toolkit, Cambridge press (United Kingdom 2004).

[13]P. C. W. Davies, S.A Fulling, W. G. Unruh, Phys. Rev. D 13 (1976) 2720

[14]J. D. Bekenstein, Phys. Rev. D 9 (1974) 12.

[15]N.Pidokrajt, Black Hole Thermodynamics, Master's Thesis in Theoretical Physics. (Stockholm University, 2003). 